D.O.I.: $10.3895 / \mathrm{S} 1808-04482006000400012$

\title{
APRESENTAÇÃO DE UM JOGO DIDÁTICO COMO FERRAMENTA DE APOIO AO ENSINO DA PRODUÇÃO ENXUTA
}

\section{PRESENTATION OF A DIDACTIC GAME AS AN AUXILIARY TOOL FOR TEACHING LEARN PRODUCTION}

\author{
Marcelo D. Depexe ${ }^{1}$; Juliana Bonacorso Dorneles ${ }^{2}$; Adolfo C. Figueiredo Costa ${ }^{3}$; Débora de Gois Santos ${ }^{4}$; \\ Luiz Fernando M. Heineck ${ }^{5}$ \\ ${ }^{1}$ Universidade Federal de Santa Catarina - UFSC - Florianópolis - Brasil marcelodepexe@ yahoo.com.br \\ ${ }^{2}$ Universidade Federal de Santa Catarina - UFSC - Florianópolis - Brasil jubd@ @otmail.com \\ ${ }^{3}$ Universidade Federal de Santa Catarina - UFSC - Florianópolis - Brasil adolfotcc@vcnet.com.br \\ ${ }^{4}$ Universidade Federal de Sergipe - UFS - Sergipe - Brasil deboragois@ yahoo.com.br \\ ${ }^{5}$ Universidade Federal de Santa Catarina - UFSC - Florianópolis - Brasil heineck@eps.ufsc.br
}

\begin{abstract}
Resumo
A utilização de jogos e simulações tem se difundido como ferramenta de apoio ao ensino na última década. O presente trabalho apresenta um jogo didático no qual se realizam simulações para a montagem de carrinhos Lego System ${ }^{\circledR}$. São abordadas diferentes formas de organização do processo produtivo, o que permite a visualização, por parte dos alunos, de diversos conceitos relativos à produção enxuta em uma situação prática. Como resultado, se obtém a redução do tempo de produção através da avaliação de elementos tais como a distribuição das atividades entre os postos de trabalho, a aplicabilidade de elementos pré-fabricados, a redução de estoques e a redução da área de trabalho. Observa-se que este método de ensino, por meio da realização de simulações, facilita a compreensão de novos conceitos, a exemplo da produção enxuta.
\end{abstract}

Palavras-chave: simulação, produção enxuta, aprendizado.

\section{Introdução}

A utilização de jogos e simulações tem se difundido como ferramenta de apoio ao ensino na última década. O uso de jogos didáticos em equipe é uma alternativa de ensino, pois permite abordar estas técnicas por meio da simulação do processo produtivo, com o objetivo de melhorar a produtividade da equipe. A vantagem do uso de simulações é que elementos do mundo real são simplificados de modo a ser possível levá-los para a sala de aula. Os jogos e simulações são considerados ferramentas pedagógicas eficientes para o ensino, pois permitem explorar estratégias alternativas e suas conseqüências, em um ambiente seguro e próximo do real, afirmam Walters, Coalter e Rasheed (1997), que avaliam o desempenho de 80 alunos em um jogo da área de administração de empresas. 
Rauch-Geelhaar, Jenke e Thurnes (2003) avaliam a utilização de jogos e simulações como instrumentos de auxílio no treinamento industrial, de modo a formar as competências necessárias onde o conhecimento atual não é suficiente. Assim, tais ferramentas de treinamento devem ser adaptadas de acordo com a demanda específica de conhecimento. Os autores salientam que apenas a combinação de diferentes formas de ensino, métodos e ferramentas é que possibilita a aquisição de competências.

Paxton (2003), por exemplo, utiliza jogos Lego System ${ }^{\circledR}$ para demonstrar a alunos de graduação e pós-graduação os conceitos relativos à curva de aprendizado, por meio de uma situação real. Deste modo, os alunos determinam sua própria curva de aprendizagem, o que facilita a assimilação do conceito em relação às tradicionais aulas expositivas. Doyle e Brown (2000) simulam condições de competitividade o mais próximas possível da realidade e verificam que as soluções geradas e os conceitos aprendidos podem ser levados para o ambiente de negócios fora da sala de aula. As habilidades desenvolvidas incluem gestão do tempo, desenvolvimento de estratégias, construção de times e capacidade de negociação.

Os blocos de montar Lego ${ }^{\circledR}$ também são utilizados em treinamento de executivos, conforme apresentado por Roos, Victor e Statler (2004), para o desenvolvimento de novas estratégias. Segundo os autores, esta é uma forma de ativar a imaginação, integrar as dimensões de experiência cognitiva, social e emocional. Os participantes utilizam o Lego ${ }^{\circledR}$ para representar e discutir questões estratégicas, organizacionais e ambientais, de modo a gerar novas idéias a respeito dos diferentes elementos do conteúdo das estratégias. De maneira similar, Bürgi, Victor e Lentz (2004) também apresentam o uso do Lego ${ }^{\circledR}$ para o desenvolvimento de estratégias em uma empresa do setor químico. Este método auxilia os gerentes a conhecer melhor seu negócio, de modo a prepara-los para reagir a mudanças repentinas.

Sun (1998) apresenta um jogo que simula de maneira simples um sistema de produção de veículos. O objetivo do jogo é explorar os conceitos de just-in-time (JIT) e material requeriment planning (MRP) por meio de uma simulação. O autor utiliza apenas papel e cartas de baralho para representar os carros e o sistema kanban utilizado na linha de montagem. Já Ammar e Wright (1999) descrevem oito atividades realizadas em sala de aula, relacionadas ao ensino na área de gestão de operações. Dentre os exemplos citados, há jogos que utilizam peças físicas, como Lego System $^{\circledR}$, além de simulações computacionais. Os autores salientam que os jogos possuem elementos de trabalho em equipe, competição e diversão, o que contribui para aumentar o entusiasmo, envolvimento e interesse dos alunos sobre os conceitos apresentados.

As diferenças entre os sistemas de produção puxado e empurrado, além dos conceitos relativos a just-in-time e kanban, são abordadas por Silveira et al. (2005), por meio de um jogo que simula uma fábrica de canetas. Os autores realizam a simulação do processo de montagem do 
produto, de modo a comparar o desempenho do grupo em diferentes formas de organização do processo produtivo. Assim, o exercício prático permite aos alunos uma melhor compreensão dos conceitos de gestão da produção. Souza e Silva et al. (2003) também apresentam um jogo de montagem de canetas, com quatro situações diferentes, de modo a abordar conceitos relativos a sistema de produção empurrado, sistema de produção puxado, além de outros conceitos, como variabilidade, redução do tempo de ciclo, simplificação, transparência, flexibilidade e balanceamento das atividades.

Neste contexto, o objetivo deste artigo é apresentar um jogo didático como ferramenta de apoio ao ensino da produção enxuta, por meio de uma simulação de uma linha de montagem de automóveis, a partir do qual se podem abordar diversos conceitos relativos aos sistemas de produção.

\section{Método de trabalho}

O jogo em questão consiste da realização de simulações para a montagem de 39 carrinhos Lego System ${ }^{\circledR}$. Os carrinhos têm o formato de carros de corrida e incluem o piloto. Para montagem de um carrinho são necessárias 18 peças: 1 capacete, 1 cabeça, 1 camisa, 1 calça, 4 rodas, 1 banco, 2 eixos, 1 chassi, 1 bloco do motor, 1 direção, 2 tanques de combustível, 1 pára-choque e 1 capô.

O estoque no posto de trabalho é representado por tapetes. Foi estipulado que o valor de cada tapete é proporcional a sua dimensão em minutos. Assim, um tapete com área correspondente a uma folha A4 vale 20 minutos; um A5, 10 minutos; um A6, 5 minutos e assim sucessivamente. O objetivo é utilizar o tapete de menor dimensão possível, de modo a induzir a produção contínua, quase que passando da mão de um operário diretamente para a mão de outro.

Estudou-se o fornecimento de materiais, com relação às distâncias de transporte e ao tamanho dos lotes, a aplicação dos conceitos da Produção Enxuta neste tipo de simulação e ainda a viabilidade de pré-fabricação de partes do carrinho. Deste modo, as regras do jogo são:

- Montagem de 39 carrinhos Lego;

- Distribuição da produção em 3 postos de trabalho;

- Cada posto possui 3 áreas de trabalho, uma para montagem, outra para recepção do carro em processo e uma terceira para estoque;

- As áreas de trabalhos são limitadas por tapetes;

- Simulação do fornecedor de materiais;

- Trabalho com estoques pequenos na produção;

- Aplicação dos conceitos da Produção Enxuta;

- Cada peça pré-fabricada custa 3 segundos. 
Os conceitos da Produção Enxuta abordados foram: simplificação das operações; compressão do ciclo de tempo; lotes pequenos; preparação do trabalho; pacotização do trabalho; padronização do trabalho; identificação de sequiências no espaço; aumento da velocidade de trabalho e seu conseqüente ritmo; paralelismo das operações; redução do tempo de atravessamento; diminuição do espaço de transporte; auto-regulação do trabalho pelos operários; efeito aprendizagem e definição do caminho crítico.

Na figura 1 é apresentado o layout da produção dos carrinhos, onde pode-se identificar os três postos de trabalho, juntamente com suas áreas de trabalho internas. Para a distribuição das tarefas realizadas em cada posto foram realizadas simulações prévias. Os fornecedores só abasteciam o estoque mediante pedido do cliente e em lotes parcelados.

Figura 1: Layout de produção dos carrinhos

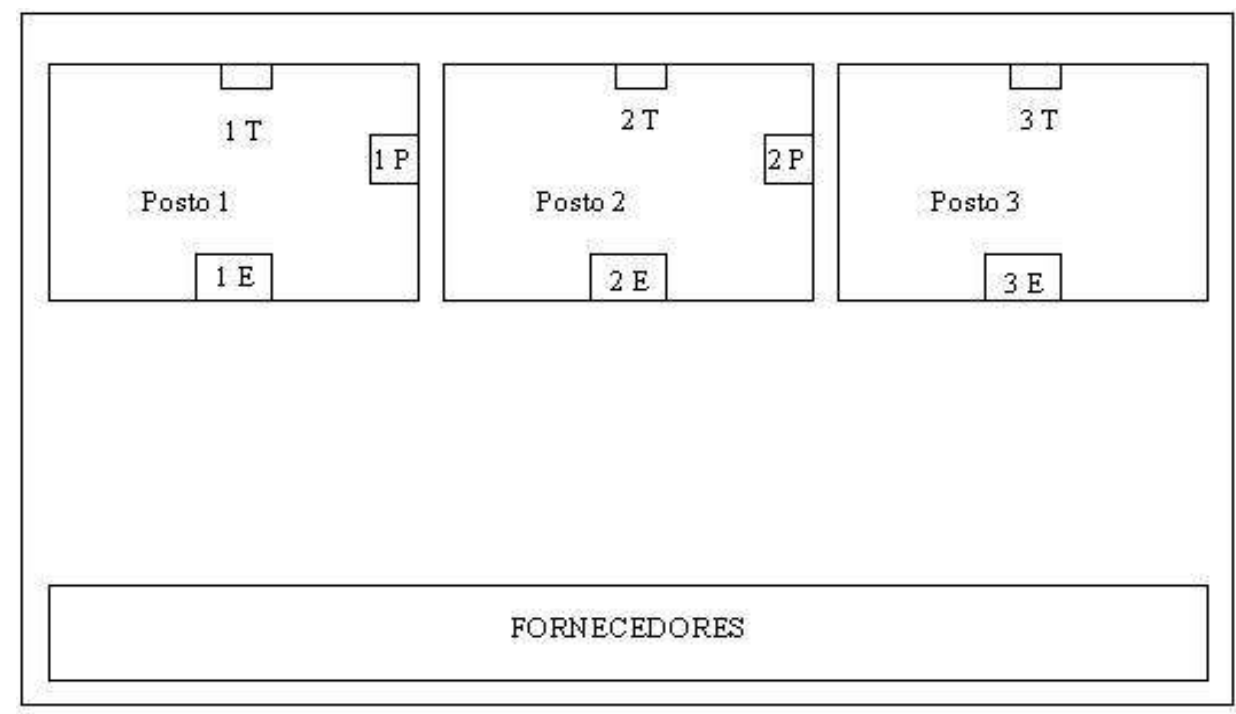

Onde: $E$ = estoque; $P$ = produção e $T=$ recep̧̧ão do carro em processo $)$

Nestas simulações, mediram-se os tempos de montagem das partes componentes e distribuíram-se os grupos de peças montadas nos postos de trabalho, de forma a considerar o balanceamento dos tempos de produção, grau de dificuldade na montagem da peça e melhor sequiência de produção. Na figura 2 (a) e (b), pode-se visualizar os produtos acabados e seus estoques iniciais, respectivamente. O manual de montagem era afixado na fábrica de modo a ser consultado pelos operadores, principalmente nas primeiras simulações para ajuste do layout e das quantidades de trabalho. Os elementos de produção eram agrupados por tipo em vasilhames plásticos, de onde eram retiradas as quantidades de materiais de acordo com o pedido. 


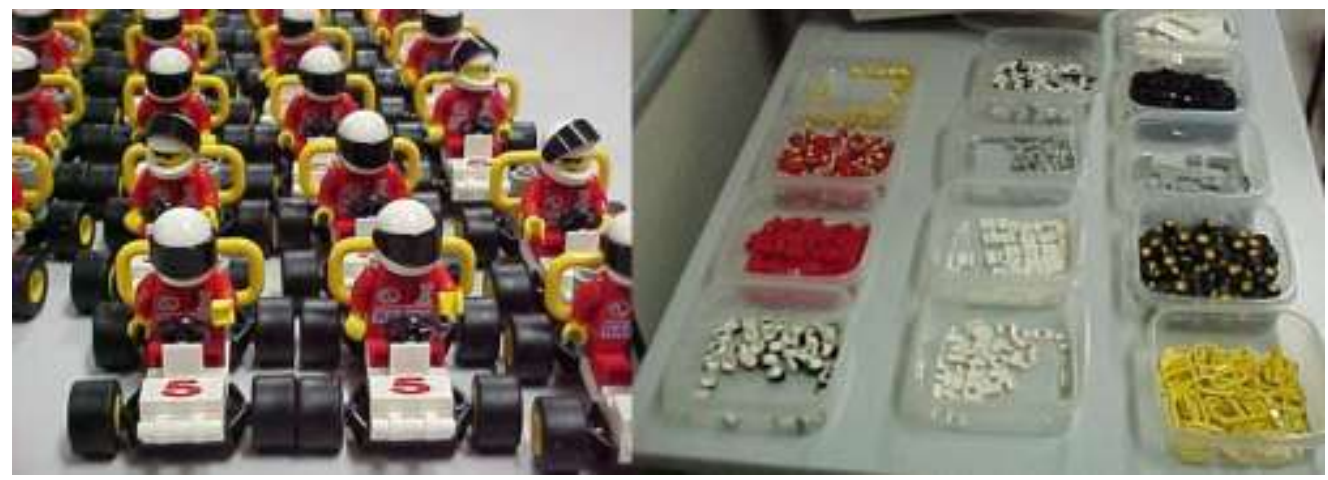

(a)

(b)

\section{Montagem dos carrinhos}

Foram realizadas três simulações de montagem dos carrinhos antes de dar início à produção em massa. A primeira simulação era composta das seguintes tarefas nos postos de trabalho:

- Posto 1: Montar o chassi, os 2 eixos, o bloco do motor, os 2 tanques de combustível e o banco. O processo levou 17 segundos para montar essas peças;

- Posto 2: Montar as 4 rodas, a direção, o pára-choque e o capô. Essa montagem resultou em 15 segundos;

- Posto 3: Montar o piloto, ou seja, o capacete, a cabeça, a calça e a camisa. Esse posto levou 6 segundos para montar as peças.

A figura 3 ilustra as peças usadas em cada posto na primeira simulação, mas ao analisar os dados, verificou-se que houve sobrecarga de trabalho nos postos 1 e 2 . Constatou-se que, no processo de montagem, o posto 2 ficou dependente do posto 1 para iniciar o seu trabalho, enquanto que no posto 3 o piloto já estava em processo de fabricação, em paralelo ao 2 .

Figura 3: Montagem nos postos de trabalho - Primeira simulação

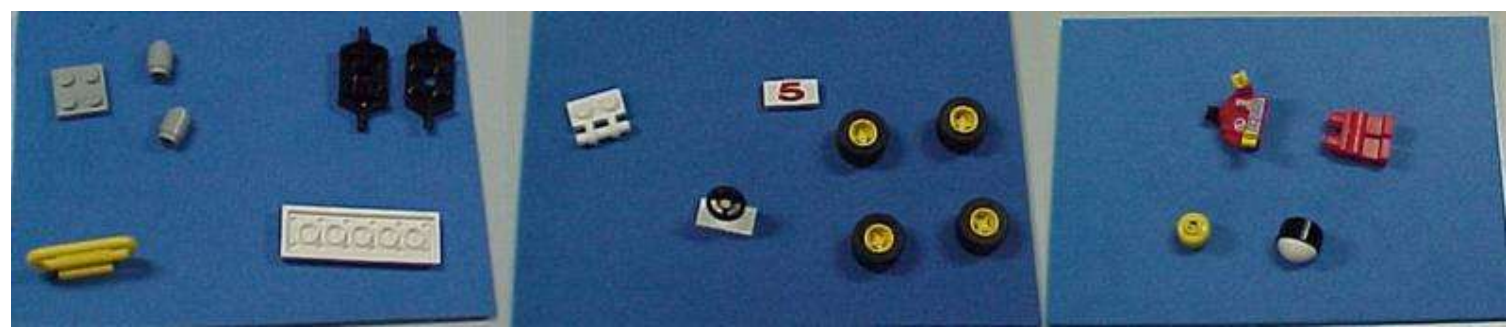

Posto 1

Posto 2

Posto 3

A mudança no processo para a nova simulação foi realizada para proporcionar o paralelismo das atividades, onde cada operário pode iniciar seu trabalho sem dependência do posto de trabalho 
anterior, ou seja, o processo pode ser iniciado e posteriormente montado no produto semi-acabado. Portanto, na segunda simulação, as tarefas em cada posto foram as seguintes:

- Posto 1: Montar o chassi, 1 eixo, 2 rodas, o capô, o pára-choque e a direção. Este posto consumiu 25 segundos;

- Posto 2: Montar 1 eixo, 2 rodas, o bloco do motor, os 2 tanques de combustível e o banco, o que levou 18,3 segundos;

- Posto 3: Não houve alteração, ou seja, o posto foi responsável pela montagem do piloto, que resultou em 6 segundos.

A figura 4 ilustra as peças usadas em cada posto na segunda simulação de montagem. Após esta primeira mudança, o posto 1 ficou sobrecarregado e o posto 3 continuou com uma carga de trabalho relativamente pequena. Cabe salientar também que, apesar das atividades poderem ser realizadas em paralelo, as medições de tempos foram independentes para cada posto, de modo a avaliar a carga de tarefa em cada posto de trabalho.

Figura 4: Montagem nos postos de trabalho - Segunda simulação

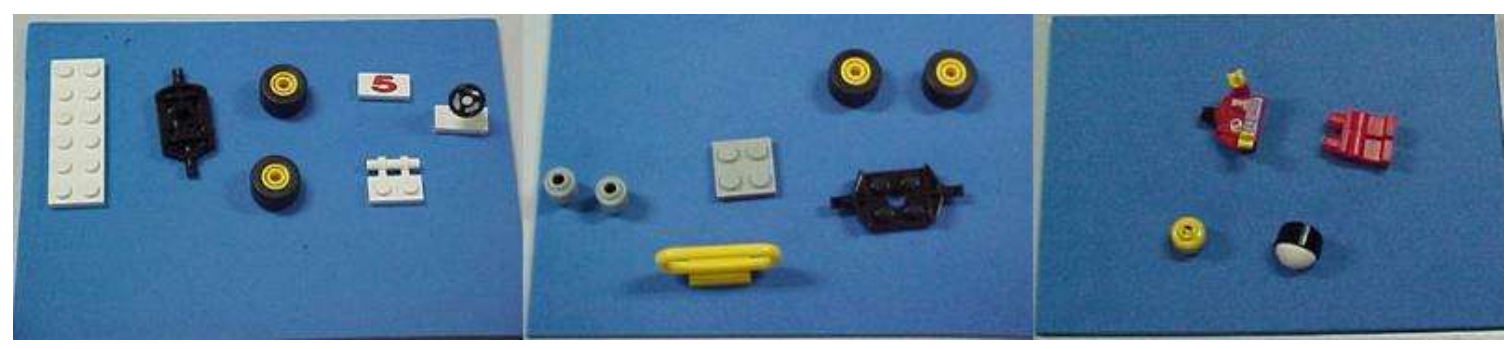

Posto 1

Posto 2

Posto 3

A mudança do processo para a terceira e última simulação teve as seguintes tarefas e tempos acumulados para a montagem de um carro em cada posto de trabalho:

- Posto 1: Montar o chassi, 1 eixo, 2 rodas, o pára-choque e a direção. Esta montagem levou 14 segundos;

- Posto 2: Montar 1 eixo, 2 rodas, o bloco do motor, os 2 tanques de combustível e o banco. Esta etapa levou 25 segundos acumulados e 9 segundos individuais;

- Posto 3: Montar o piloto (o capacete, a cabeça, a calça e a camisa) e o capô. Levou 29,51 segundos acumulados e 4,51 segundos individuais para montar essas peças.

Ao contrário das outras simulações, nesta última a medição dos tempos de montagem foi realizada considerando os tempos acumulados na linha de montagem. Desse modo, pode ser verificado como as tarefas dos postos 2 e 3 foram incorporadas dentro do tempo de montagem realizado no posto 1 , o que justifica o uso do paralelismo das tarefas. A figura 5 apresenta as peças utilizadas em cada posto na terceira simulação de montagem. 
Figura 5: Montagem nos postos de trabalho - Terceira simulação

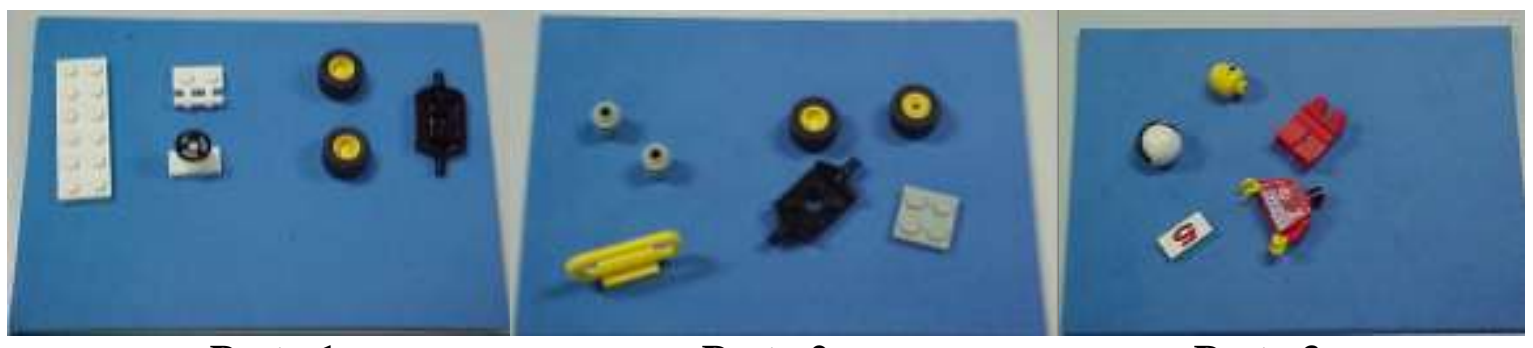

Posto 1

Posto 2

Posto 3

As decisões tomadas antes do início da produção plena e as justificativas adotadas são as seguintes:

- Necessidade de uma área de montagem para simular o espaço físico necessário no caso real. O espaço de trabalho, representado pelo tapete, é de uma área suficiente para apoiar um carro, ou seja, foi imobilizada uma área no formato A8 em cada posto de trabalho, com um tempo total de multa igual a 225 segundos;

- Estoque de 2 carrinhos em cada posto de trabalho, pois 1 carrinho necessitaria de muito transporte para o fornecimento e 3 carrinhos representaria um alto investimento no estoque inicial do processo;

- Estocagem do material em recipientes fechados lateralmente (baias), pois existiam peças pequenas que facilmente poderiam se espalhar pelo posto de trabalho. Esta decisão implicou no uso de uma área de estocagem correspondente ao tamanho do recipiente, o que imobilizou uma área no formato A6 em cada posto de trabalho e multa de 900 segundos;

- Área de processo suficiente para apoiar dois carros em processo, imobilizando uma área A7 nos postos 1 e 2 e multa de 300 segundos;

- 3 montadores, 2 fornecedores e um gerente (controle da produtividade). Esta decisão foi tomada porque um fornecedor não consegue atender a demanda de materiais, quando se trata de grande diversidade de peças;

- Controle da produção a cada lote de 4 carros, com o registro dos tempo acumulados de fabricação. A escolha do lote foi estipulada em 4 para facilitar o resgate das informações. $\mathrm{Na}$ primeira rodada foram verificados dois problemas durante a produção dos carrinhos:

- Gargalo no posto 2, dificuldade ao montar os dois tanques e o banco;

- Falha no posto 3, ao colocar o piloto, o tanque de combustível se desprendiam. 
O fato fez com que o grupo sugerisse a pré-fabricação dos tanques, banco e bloco do motor. Então foi tomada a decisão de um novo teste, onde a pré-fabricação foi inserida no processo. Como resultado obteve-se a montagem do carrinho em 21 segundos, enquanto que anteriormente o carrinho era montado em 29,51 segundos. Porém, a pré-fabricação foi inviabilizada porque a multa de 3 segundos para cada peça contida no material pré-fabricado resultava em uma penalidade total por carrinho de 12. Outro motivo que influenciou a decisão de não pré-fabricar esse conjunto de material foi a constatação que somente o problema no posto 2 seria resolvido. As decisões para melhorar a produção foram as seguintes:

- Alterar a linha de montagem, antecipar a montagem do piloto para o posto 2, e montar os tanques e o banco no posto 3;

- Reduzir as áreas de recepção (de A7 para A9) e de montagem (de A8 para A9), passando a ter uma multa de 111 e 74 segundos respectivamente, por não haver a necessidade desses acréscimos de áreas para realização das tarefas;

- Realizar novas simulações para escolha de nova linha de montagem, de modo a determinar a seqüência de montagem ideal.

Em novas simulações obteve-se, numa primeira tentativa, o tempo de 29 segundos por carrinho, não melhorando significativamente em relação ao tempo anterior. Na segunda tentativa o tempo foi reduzido em 1,50 segundos do tempo inicial. Então a nova linha de montagem teve as seguintes tarefas em cada posto de trabalho, bem como os tempos acumulados para a montagem de um carro:

- Posto 1: Montar o chassi, 2 eixo, 4 rodas, o que levou 14 segundos;

- Posto 2: Montar o piloto (capacete, a cabeça, a calça e a camisa) além da direção. Esta etapa resultou em 24 segundos acumulados e 10 segundos individual;

- Posto 3: Montar o pára-choque, o capô, o bloco do motor, os 2 tanques de combustível e o banco. A montagem neste posto levou 28 segundos acumulados e 4 segundos individual.

A figura 6 mostra as peças usadas em cada posto na terceira simulação de montagem.

Figura 6: Montagem nos postos de trabalho - Alteração do processo

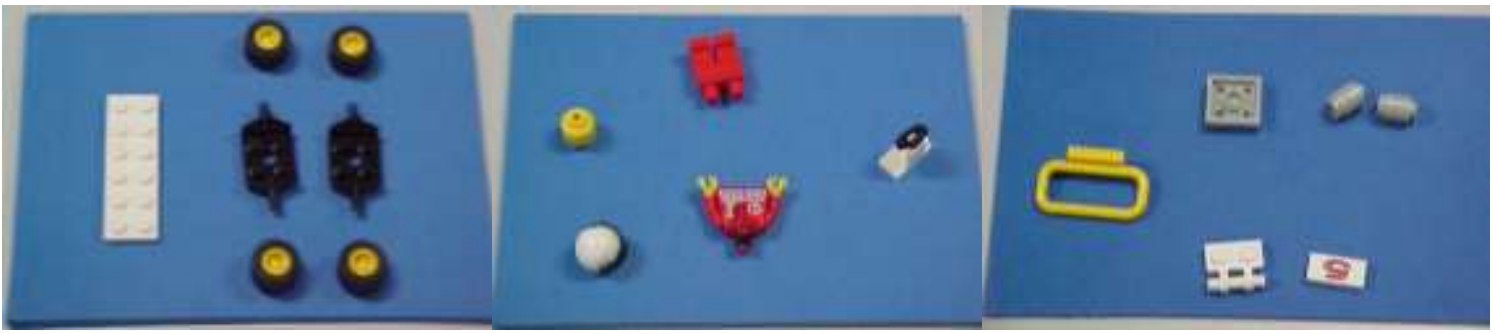

Posto 1

Posto 2

Posto 3 
A tabela 1, por sua vez, apresenta o controle de produção das duas rodadas de montagens.

Tabela 1 Controle de produção das duas rodadas de montagem

\begin{tabular}{c|c|c|c|c}
\hline \multirow{2}{*}{ Carrinhos } & \multicolumn{2}{|c|}{ PRIMEIRA RODADA } & \multicolumn{2}{c}{ SEGUNDA RODADA } \\
\cline { 2 - 5 } & Tempo Acumulado & $\begin{array}{c}\text { Tempo do } \\
\text { Ciclo }\end{array}$ & Tempo Acumulado & $\begin{array}{c}\text { Tempo do } \\
\text { Ciclo }\end{array}$ \\
\hline 4 & 101 & 101 & 85 & 85 \\
\hline 8 & 198 & 97 & 170 & 85 \\
\hline 12 & 271 & 73 & 345 & 75 \\
\hline 16 & 363 & 92 & 410 & 75 \\
\hline 20 & 468 & - & 485 & 75 \\
\hline 24 & - & - & 670 & 85 \\
\hline 28 & 542 & 88 & 720 & 80 \\
\hline 32 & 630 & 61 & 770 & 50 \\
\hline 36 & 721 & & $(1085($ MULTA)+ & \\
\hline 39 & 782 & $770 * 6) / 39=$ & \\
\hline $\begin{array}{c}\text { Total por } \\
\text { carrinho }\end{array}$ & $(1425($ MULTA) + & & 146 s/carrinhos & \\
\hline
\end{tabular}

A primeira rodada apresenta os dados obtidos segundo a sequiência de montagem apresentada na figura 5, que é um aperfeiçoamento das tentativas expressas nas figuras 3 e 4 . Já a segunda rodada utiliza a configuração apresentada na figura 6, que foi elaborada após a identificação das falhas observadas na primeira rodada. Deste modo, os alunos perceberam a influência da seqüência de montagem no tempo total de atravessamento, bem como outros conceitos relativos aos sistemas de produção.

\section{Conclusões}

O presente trabalho apresentou o relato de uma experiência na qual se utiliza um jogo para complementar o processo de aprendizado em sala de aula. A partir do jogo realizado, os alunos puderam compreender melhor os conceitos da produção enxuta, tais como simplificação das operações, tempo de ciclo, lotes pequenos, preparação do trabalho, pacotização do trabalho, padronização do trabalho, identificação de sequiências no espaço, paralelismo das operações, redução do tempo de atravessamento, diminuição do espaço de transporte, auto-regulação do trabalho pelos operários, sincronia, efeito aprendizagem e definição do caminho crítico.

Ressalta-se ainda que, mesmo que todos estes conceitos sejam compreendidos pelos alunos, se não houver um planejamento prévio efetivo, o resultado final não será positivo, ou seja, o uso adequado destes conceitos direciona a preparação para o trabalho, de modo a reduzir variabilidades e a aumentar a produtividade da equipe. Na simulação, verificou-se ainda a importância de estudos pilotos da linha de produção, com disposição de peças, relação com o fornecedor, polivalência dos participantes e balanceamento da quantidade de trabalho nos postos de produção. 
A pré-fabricação, por intermédio de penalidades na forma de multas, levou os participantes a analisarem seu custo/benefício durante as simulações, como ocorre nos casos reais, onde se paga um preço elevado para se obter maior velocidades de produção. O mais importante é que todos estes conhecimentos e a comparação entre situações diferenciadas de layout de trabalho foram viabilizados pelo jogo sem o dispêndio de muito de tempo e recursos físicos, bem como desperdício de materiais, o que seria comum em casos reais.

\begin{abstract}
The utilization of games and simulations has been diffused as an auxiliary tool for teaching on the last decade. This paper presents a didactic game that simulate the assembly line of Lego Systems ${ }^{\circledR}$ cars. It was approached different forms of production process organization, what allows the students visualize many concepts related to lean production on a practice situation. The investigation result in the production time reduced through elements evaluation, how: activities distribution between station works, used of prefabrication materials, stock reduced, and station work reduced. This method of education, by means of the simulation accomplishment, facilitates the understanding of new concepts, like the lean production.
\end{abstract}

Key-words: simulation, lean production, learning.

\title{
Referências
}

AMMAR, S. \& WRIGHT, R. Experiential learning activities in operations management. International Transactions in Operational Research, v. 6, n. 2, p. 183-197, 1999.

cross ${ }^{\text {ref }}$

BÜRGI, P.; VICTOR, B. \& LENTZ, J. Modeling how their business really works prepares managers for sudden change. Strategy \& Leadership, v. 32, n.2, p. 28-35, 2004.

cross ${ }^{\text {ref }}$

DOYLE, D. \& BROWN, F. W. Using a business simulation to teach applied skills - the benefits and the challenges of using student teams from multiple countries. Journal of European Industrial Training, v. 24, n. 6, p. 330-336, 2000.

cross ${ }^{\text {ref }}$

PAXTON, J. Teaching brief: a short, simple learning curve classroom exercise. Decision Sciences Journal of Innovative Education, v. 1, n. 2, p. 303-307, 2003.

cross ${ }^{\text {ref }}$

RAUCH-GEELHAAR, C.; JENKE, K.; THURNES, C. M. Gaming in industrial management - quality and competence in advanced training. Production Planning \& Control, v. 14, n. 2, p. 155-165, 2003.

cross ${ }^{\text {ref }}$

ROOS, J.; VICTOR, B. \& STATLER, M. Playing seriously with strategy. Long Range Planning, v. 37, p. 549-568, 2004.

cross ${ }^{\text {ref }}$ 
SILVEIRA, J. P.; DEPEXE, M. D.; GASPARETTO, F. C.; DORNELES, J. B.; SANTOS, D. G. \& HEINECK, L. F. M. Fábrica de canetas - aprendendo conceitos de produção a partir de jogos em equipe. In: SIMPÓSIO BRASILEIRO DE GESTÃO E ECONOMIA DA CONSTRUÇÃO, IV, 2005, Porto Alegre. Anais... Porto Alegre-RS, 2005,9 p.

SOUZA e SILVA, M. F.; BRESSIANI, L.; SAFFARO, F. A.; SANTOS, D. G. \& HEINECK, L. F. M. Sistema de produção puxado e sistema de produção empurrado: simulação através de jogo didático de montagem de canetas, associando idéias e conceitos ao ambiente da construção civil. In: SIMPÓSIO BRASILEIRO DE GESTÃO E ECONOMIA DA CONSTRUÇÃO, III, 2003, São Carlos. Anais... São Carlos-SP, 2003, 11 p.

SUN, H. A game for the education and training of production/operations management. Education + Training, v. 40, n. 9, p. 411-416, 1998 .

WALTERS, B. A.; COALTER, T. M. \& RASHEED, A. M. A. Simulation games in business policy courses: is there value for students? Journal of Education for Business, v. 72, n. 3, p. 170-174, 1997.

cross ${ }^{\text {ref }}$

\section{Dados dos autores:}

Marcelo Dalcul Depexe

Universidade Federal de Santa Catarina - UFSC

Programa de Pós-Graduação em Engenharia de Produção - PPGEP

Rua Conselheiro Laurindo, 557, ap 306. Curitiba/PR - Brasil. CEP 80060-100

Telefones: (41) 3330-7218

e-mail: marcelodepexe@yahoo.com.br

Juliana Bonacorso Dorneles

Universidade Federal de Santa Catarina - UFSC

Programa de Pós-Graduação em Engenharia Civil - PPGEC - Doutoranda

Rua João Pio Duarte Silva, 404, Condomínio Liberty Park, Edifício Juriti, apto 203, Bairro Córrego

Grande, Florianópolis/SC - Brasil. CEP 88037-000

Telefones:(48) 3331-5197-UFSC(GDA)/3025-1437-residencial/8411-0434

e-mail: jubd@hotmail.com

Adolfo Cesar Figueiredo Costa

Universidade Federal de Santa Catarina - UFSC

Programa de Pós-Graduação em Engenharia Civil - PPGEC - Mestrando

Rua Lauro Linhares, 689, Cond. Granville, ap 303 B9, Bairro Trindade, Florianópolis/SC - Brasil.

CEP 88036-000

Telefone: (48) 8403-8062

e-mail: adolfocfc@gmail.com 
Débora de Gois Santos

Universidade Federal de Sergipe - UFS

Departamento de Engenharia Civil - Professor Adjunto

Avenida Desembargador Maynard, 1005. Bairro Cirurgia. Aracaju/SE - Brasil. CEP 49050-210

Telefones: (79) 9998 8216; (79) 32126704 (UFS); (79) 32244573 (residência)

e-mail: deboragois@ufs.br

Luiz Fernando M. Heineck

Universidade Federal de Santa Catarina - UFSC

Programa de Pós-Graduação em Engenharia de Produção - PPGEP

Professor Titular

CTC/UFSC Cx. postal 476. Florianópolis/SC - Brasil. CEP 88010-970

e-mail: heineck@eps.ufsc.br

Recebido para publicação em: 18/10/2006

Aceito para publicação em: 24/11/2006 